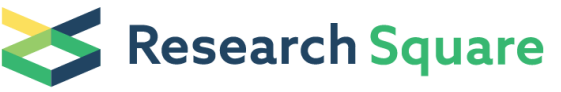 \\ Preprints are preliminary reports that have not undergone peer review. \\ They should not be considered conclusive, used to inform clinical practice, or referenced by the media as validated information.
}

\section{Vascular Access for Lipid Apheresis: A Challenge in Young Children With Homozygous Familial Hypercholesterolemia}

Julia Lischka

Medical University of Vienna

Klaus Arbeiter

Medical University of Vienna

Charlotte de Gier

Medical University of Vienna

Andrea Willfort-Ehringer

Medical University of Vienna

Nina-Katharina Walleczek

Medical University of Vienna

Renata Gellai

Medical University of Vienna

Michael Boehm

Medical University of Vienna

Albert Wiegman

University of Amsterdam, Academic Medical Center

Susanne Greber-Platzer ( $\nabla$ susanne.greber-platzer@meduniwien.ac.at )

Medical University of Vienna

\section{Research Article}

Keywords: vascular access, children, pediatric, LDL-cholesterol, lipid apheresis, arteriovenous fistula

Posted Date: April 14th, 2021

DOI: https://doi.org/10.21203/rs.3.rs-405265/v1

License: (1) (i) This work is licensed under a Creative Commons Attribution 4.0 International License.

Read Full License

Version of Record: A version of this preprint was published at BMC Pediatrics on March 12th, 2022. See the published version at https://doi.org/10.1186/s12887-022-03192-7. 


\section{Abstract}

\section{Background}

Homozygous familial hypercholesterolemia $(\mathrm{HoFH})$ is a rare genetic disorder leading to extremely increased LDL-cholesterol (LDL-C), resulting in high cardiovascular risk in early childhood. Lipid apheresis (LA) is an effective treatment and should be started as early as possible to prevent premature cardiovascular events. As peripheral punctures in children can be challenging due to small vessels and anxiety, this study aimed to evaluate central venous catheters (CVCs) as vascular access for LA in young children with hoFH.

\section{Methods}

Retrospective analysis (2016-2019) on four children with hoFH aged 3-5, performing weekly or biweekly LA with a CVC.

\section{Results}

LDL-C decreased by $>60 \%$. In three children, the use of a permanent CVC for 698,595 , and 411 days, respectively, avoided difficult peripheral access, without the occurrence of occlusion or thrombosis. Unfortunately, one child had recurrent CVC-related infections and needed an arteriovenous fistula from the age of 5 .

Although the mean dwell time per catheter was 212 days, there were, as expected, severe side effects of early catheter infections with sepsis and accidental self-removal. Starting LA at an early age improved or stabilized carotid intima-media thickness (IMT) in all children. Finally, IMT did increase in one child caused by intolerance to peripheral punctures and LA interruption.

\section{Conclusions}

Permanent CVCs are a viable temporary access choice for LA in young children with hoFH until peripheral venipuncture is practicable. The risk of CVC-related infections needs to be taken into account.

\section{Background}

Homozygous familial hypercholesterolemia (hoFH) is a rare autosomal-dominant genetic disorder with an estimated prevalence of 1:160,000-300,000 [1]. It leads to a significant increase in LDL cholesterol (LDL-C) from birth onwards (above $500 \mathrm{mg} / \mathrm{dL}$ ), resulting in high cardiovascular risk in early childhood. Hence, the earliest initiation of lipid-lowering treatment is required to prevent premature cardiovascular damage [1-4].

Fat-modified diets and lipid-lowering medications are hoFH treatment strategies, with minimal success [1, $2,5]$. Therefore, lipoprotein apheresis (LA) plus statins- ezetimibe combination therapy has become 
standard practice to reduce LDL-C levels [6]. Expert consensus recommends reducing LDL-C levels by $50 \%$ in heterozygous $\mathrm{FH}$ children, with a target $\mathrm{LDL}-\mathrm{C}<130 \mathrm{mg} / \mathrm{dL}$ from 10 years onwards. There are no clear recommendations for younger children [1].

Children suffering from hoFH are at very high risk for cardiovascular events, with reported cases of 3- to 4 -year-olds manifesting angina pectoris and even fatal myocardial infarctions [7-10]. As untreated hoFH patients have a life expectancy of less than 30 years [2], Guidelines recommend starting LA as early as possible $[2,10]$, preferably at age $2[1]$, to decrease the risk of cardiovascular diseases and prolong life expectancy.

LA is a highly effective method to reduce LDL-C with an expected mean reduction of up to $80 \%$ after a single treatment [11]. Venous access is needed for weekly or biweekly LA procedures [12]. However, repeated peripheral punctures to achieve an adequate blood flow is often challenging in young children due to small peripheral vessels and severe anxiety $[6,13,14]$. Permanent central venous catheters (CVCs) or arteriovenous fistulas (AVF) are possible alternatives and usually optimize treatment. Moreover, CVCs allow children to move their arms and body, thus being better tolerated[1, 2, 5, 14].

While vascular access options for hemodialysis have been extensively reviewed $[13,15,16]$, there is limited evidence on surgical venous access in LA [17-19]. Only a few studies reported on

hypercholesterolemic patients [20], one case study [21], and one recently published systematic review [14] investigated LA in children with hoFH specifically. However, research on venous access techniques in pediatric patients is still lacking, making an evidence-based approach difficult.

The aim of this study was to evaluate central venous catheters as a possible venous access for regular LA in young children with hoFH.

\section{Methods}

The study includes four young children (aged 2-5 years) with genetically confirmed homozygous familial hypercholesterolemia from the Outpatient Clinic for Pediatric Obesity and Dyslipidemia of the Department of Pediatrics and Adolescent Medicine at the Medical University of Vienna, who underwent LA between October 2016 and November 2019.

LA, which selectively removes apolipoprotein B (ApoB) 100-containing lipoproteins from the patients' blood via extracorporeal filtration, was performed weekly or biweekly. A Therasorb column by Miltenyi Biotec was used with a plasma Adasorb filled with $5 \%$ albumin and a volume of $100 \mathrm{ml}$. Systemic anticoagulation consisted of bolus administration of citrate and heparin, followed by a continuous citrate/heparin infusion.

Permanent (tunneled) CVCs (Permcath, Quinton Instrument Co., Seattle, USA or Broviac, BARD Access Systems, Salt Lake City, USA) were surgically implanted in the subclavian or jugular veins. The CVC lines were filled with an alteplase solution in interval periods. 
All four study participants underwent physical examinations, and medical history, clinical and laboratory data were collected.

Lipid levels, physical and additional clinical examinations like electrocardiogram (ECG), echocardiography, supra-aortic intima-media thickness (IMT), and coronary CT / coronary catheter were analyzed at baseline, during $L A$, and at least after three years (up to 1,138 days) of LA.

IMT was measured by carotid ultrasound at the Division of Angiology, Department of Internal Medicine at the Medical University of Vienna. Mean IMT was calculated out of 12 consecutive measurements per each side. The clinical assessment referred to age- and sex-specific reference values based on internal data (unpublished).

CVC-related complications were classified into six subgroups according to the literature: mechanical (dislocation, migration, rupture, self-removal by accident) [22-24], malfunction/occlusion (except thrombosis) [22, 23, 25], thrombotic (thrombosis, embolism, deep vein thrombosis) [22, 23], infectious (local vs. systemic; CRBSI: catheter-related bloodstream infection) [22, 23, 25], complications associated with implantation (pneumo-, hydro-, hemothorax, arterial puncture, cardiac tamponade, arrhythmia, bleeding, air embolism, vessel perforation, hematoma) [22, 24], and not otherwise classified complications e.g., loss of central venous patency after multiple CVC insertions [26, 27].

\section{Patients}

LA was started at the age of 3 to 5 years in four children with severe hoFH (molecularly proven and LDL-c $>500 \mathrm{mg} / \mathrm{dL}$ after diet and lipid-lowering medication). Statins (simvastatin or atorvastatin, $2 \mathrm{mg} / \mathrm{kg}$, max. $40 \mathrm{mg} / \mathrm{d})$, ezetimibe (10 mg), and colesevelam ( $625 \mathrm{mg} 2 \mathrm{x} /$ day) were administered orally. Patient 1 refused colesevelam because of abdominal side effects.

\section{Statistics}

Due to the small sample size, descriptive statistics were used to analyze the data. Categorical data were analyzed using absolute frequencies and relative frequencies.

All statistical analyses were performed using Microsoft Excel 2010 for Windows 7 (Microsoft Corp., Redmond, WA, USA).

\section{Ethics}

The study protocol was approved by the ethics committee of the Medical University of Vienna (No. 1638/2019) and conforms to the Helsinki declaration guidelines.

\section{Results}


Table 1

Patient characteristics

\begin{tabular}{|llllllll|}
\hline $\begin{array}{l}\text { Patient } \\
(\text { No.) }\end{array}$ & $\begin{array}{l}\text { Sex } \\
(\mathrm{w} / \mathrm{m})\end{array}$ & $\begin{array}{l}\text { Genetic } \\
\text { mutation }\end{array}$ & $\begin{array}{l}\text { Age at } \\
\text { diagnosis } \\
\text { (years) }\end{array}$ & $\begin{array}{l}\text { Period } \\
\text { of } \\
\text { CVC } \\
\text { use } \\
\text { (age in } \\
\text { years) }\end{array}$ & $\begin{array}{l}\text { Cumulative } \\
\text { months of } \\
\text { CVC use }\end{array}$ & $\begin{array}{l}\text { Age at } \\
\text { follow-up } \\
\text { (years) }\end{array}$ & $\begin{array}{l}\text { Venous } \\
\text { access at } \\
\text { follow-up }\end{array}$ \\
\hline 1 & w & $\begin{array}{l}\text { LDLR } \\
\text { homozygous }\end{array}$ & 3 & 5 to 7 & 24 & 8 & peripheral \\
\hline 2 & w & $\begin{array}{l}\text { LDLR } \\
\text { homozygous }\end{array}$ & 2 & 4 to 6 & 23 & 7 & peripheral \\
\hline 3 & w & $\begin{array}{l}\text { LDLR } \\
\text { homozygous }\end{array}$ & 2 & 5 to 7 & 30 & 8 & peripheral \\
\hline 4 & m & $\begin{array}{llllll}\text { LDLR } \\
\text { homozygous }\end{array}$ & 1 & 3 to 5 & 8 & 7 & AVF \\
\hline
\end{tabular}

Initially, the permanent CVCs were surgically implanted in four children with hoFH (three girls, one boy) aged 3 to 5 . All patients started with biweekly LA, later intensified to weekly intervals for three of them. Patient 4 had several CVC complications and therefore received an AVF eight months later, at the age of 5 years. The venous situation and tolerance to peripheral venous punctures progressively improved in the three female patients; therefore, CVCs could be removed at the age of 6 years in one girl and 7 years in the other two. (Table 1).

All four children needed iron supplementation throughout LA, indicating chronic iron deficiency anemia.

Mean baseline LDL-C levels with a maximum dose of lipid-lowering medication before the start of LA ranged from 535 to $742 \mathrm{mg} / \mathrm{dL}$. With LA, mean LDL-C levels decreased to 244 up to $411 \mathrm{mg} / \mathrm{dL}$ pre-LA, and 76 up to $154 \mathrm{mg} / \mathrm{dL}$ post-LA (Table 2). Thus, LA reduced LDL-C levels by more than $60 \%$ in all four children. As a positive side effect, LA also decreased Lipoprotein (a), if elevated (not shown).

In three children undergoing weekly LA procedures, the pre-LA LDL-C remained high one week after LA, with values between 252 to $411 \mathrm{mg} / \mathrm{d}$, whereas one girl continued biweekly sessions, with pre-LA LDL-C values of around $244 \mathrm{mg} / \mathrm{dL}$ two weeks after LA (Table 2). 
Table 2

LA treatment: Lipid levels at baseline and after 3 years of LA.

\begin{tabular}{|c|c|c|c|}
\hline \multirow[t]{2}{*}{ Pat. } & \multirow[t]{2}{*}{ LDL-C [mg/dL] on max. oral medication } & \multirow{2}{*}{$\begin{array}{l}\text { Steady state Pre-LA } \\
\text { LDL-C [mg/dL] }\end{array}$} & \multirow{2}{*}{$\begin{array}{l}\text { Steady state Post-LA } \\
\text { LDL-C [mg/dL] }\end{array}$} \\
\hline & & & \\
\hline \multirow[t]{2}{*}{1} & $688.8^{\mathrm{A}}$ & 411.2 & 153.5 \\
\hline & & $(288.8-564.6)$ & $(113.8-227.8)$ \\
\hline \multirow[t]{2}{*}{2} & 535.4 & 337.2 & 104.7 \\
\hline & $(534.8-536.0)$ & $(265.0-443.0)$ & $(84.8-151.6)$ \\
\hline \multirow[t]{2}{*}{3} & 597.1 & $244.2^{\mathrm{B}}$ & $81.0^{\mathrm{B}}$ \\
\hline & $(521.2-732.0)$ & $(223.0-287.4)$ & $(67.4-99.0)$ \\
\hline \multirow[t]{2}{*}{4} & $742.0^{\mathrm{A}}$ & 251.7 & 76.4 \\
\hline & & $(205.0-297.4)$ & $(57.2-94.8)$ \\
\hline \multicolumn{4}{|c|}{ Values are displayed as means, minimum and maximum over a period of 3 months } \\
\hline \multicolumn{4}{|c|}{ A only one measurement available } \\
\hline \multicolumn{4}{|c|}{ Bbiweekly } \\
\hline
\end{tabular}

Values at baseline are on maximum oral medication with statins and ezetimibe in all patients and colesevelam in patient 2. Lipid values were measured before and after weekly/biweekly LA.

Between October 2016 and November 2019, the four patients had a total of 106, 104, 75, and 94 LA sessions, respectively. In patient 1 , starting $L A$ aged 5 , the critical peripheral venous situation required a CVC implantation due to high blood flow. Infections and accidental self-removal of the CVCs occurred five times, and in between, several unsuccessful venous punctures were attempted, which were painful and stressful for the child, despite extensive psychological support. Nine LA sessions were missed, and four were interrupted. The implantation of a new CVC stabilized LDL-C values.

After three years of LA treatment, skin xanthomas decreased or disappeared in all patients. (In detail, patient 1 showed a remission of the xanthoma on the elbows, knees, and upper legs. In patient 2, the xanthoma on both Achilles tendons, knees, and dorsum of both feet nearly disappeared except for small residues on the left knee and right Achilles tendon. In patient 3, the xanthoma on hands, knees, and dorsum of both feet decreased, and the arcus lipoides diminished. Patient 4 showed complete remission of the xanthoma on both Achilles tendons, right knee, and left foot dorsum.)

Pre-treatment echocardiography revealed mild aortic insufficiency in two children, while another child had mild aortic insufficiency at 3-year follow-up. Three children showed increased age- and gender-specific baseline carotid IMT levels, which remained unchanged, decreased, or normalized after three years of LA treatment. Patient 1 developed a new vascular plaque formation in the left external carotid artery, without 
hemodynamic significance. Before starting LA, a coronary CT angiography performed on all patients revealed normal coronary arteries without plaque formation or stenosis.

Table 3

CVC complications

\begin{tabular}{|lll|}
\hline Complications & $\begin{array}{l}\text { Number } \\
\text { total }\end{array}$ & $\begin{array}{l}\text { Number of } \\
\text { patients } \\
\text { with at least 1 } \\
\text { event }\end{array}$ \\
\hline Infections & 9 & 4 \\
\hline -) Systemic infection: Sepsis/SIRS & 3 & 2 \\
\hline -) Systemic infection: CRBSI & 4 & 2 \\
\hline -) Local CVC-infection & 2 & 1 \\
\hline Mechanical & 9 & 3 \\
\hline -) Self-removal by accident & 6 & 3 \\
\hline -) Dislocation & 3 & 2 \\
\hline Malfunction: occlusion & 0 & 0 \\
\hline Thrombosis & 0 & 0 \\
\hline $\begin{array}{l}\text { Complications associated with } \\
\text { implantation }\end{array}$ & 0 & 0 \\
\hline Other. Pneumo-Mediastinum/Pericard & $\mathbf{1}$ & $\mathbf{1}$ \\
\hline
\end{tabular}

Different subgroups defined as CVC related complications. Each patient suffered from at least one infection, in 3 cases sepsis occurred. The most frequent complication was accidental self-removal of the catheter. CRBSI: Catheter-Related Blood Stream Infection

Complications associated with CVCs are listed in Table 3. Infections and mechanical complications occurred most frequently. All four patients suffered from a bacterial infection progressing to bacterial sepsis in three instances (Table 3): Staphylococcus aureus in five cases and Plesiomonas shigelloides in one.

Patient 1 was hospitalized twice for 13 and 15 days, respectively, due to catheter-related sepsis caused by Staphylococcus aureus, and each time a new CVC had to be implanted. The family excluded the option to place an AVF. Thus, patient 1 had a total of four CVC replacements over a 3-year period (Table 4).

Patient 2, who presented with catheter related complications twice, had an CRBSI event that resolved without further issues. Plesiomonas shigelloides was identified in the blood culture. 
Patient 3 experienced one severe complication shortly after the first CVC implantation and had to be hospitalized with Systemic Inflammatory Response Syndrome that later induced Staphylococcal Scalded Skin Syndrome.

Patient 4 had multiple catheter-related complications in the first year of LA: four CVC dislocations, leading to catheter loss twice, and three episodes of CRBSI requiring hospitalization. Therefore, he received an AVF eight months later (Table 4).

Mechanical complications such as accidental self-removal and dislocation of the CVCs occurred in three children. Patient 1 needed five CVCs in total (Table 4).

In two girls, patients 2 and 3, CVCs were stable and could be used for 595 days (19 months) and 698 days (23 months), respectively (Table 4). After accidental self-removal in patient 2 at the age of 6 , the switch to peripheral punctures succeeded, thanks to the emotional support given by the child's mother. Peripheral puncture attempts in patient 3 at the age of 7 were less successful due to anxiety, stress, and psychological pressure, requiring implantation of a new CVC 1.5 months later.

Aged 6, patient 1 referred thoracic pain and dyspnea suddenly after a ball shot to the upper thorax during school sports. Small pneumomediastinum and pneumopericardium were diagnosed, and the girl was hospitalized for observation. The CVC was still intact, and the air was rapidly absorbed without further complications.

No other catheter-related complications like occlusion, venous thrombosis, or perioperative problems were observed (Table 3). Ultrasound measurement always showed normal central veins anatomy and blood flow. 
Table 4

Site of venous access, dwell time and reason for removal of CVC per patient in chronological order

\begin{tabular}{|llll|}
\hline Patient (No.) & Site of venous access & dwell time in days & reason for removal \\
\hline & & \\
\hline V. jug. sin. & 19 & Sepsis \\
\hline V. subcl. sin. & 411 & Sepsis, Self-removal by accident \\
\hline V. jug. sin. & 51 & Self-removal by accident \\
\hline V. subcl. dex. & 66 & Self-removal by accident \\
\hline V. subcl. sin. & 183 & Dislocation, local infection \\
\hline Peripheral access & at the age of 7 years & \\
\hline
\end{tabular}

2

V. subcl. sin. $698 \quad$ Self-removal by accident

Peripheral access at the age of 6 years

3

V. jug. sin. $14 \quad$ Sepsis

V. subcl. sin. $\quad 595 \quad$ Elective removal

V. subcl. dex. $\quad 280 \quad$ Elective removal

Peripheral access at the age of 7 years

4

V. jug. sin. $\quad 71$

$71 \quad$ Self-removal by accident

V. subcl. sin. $\quad 55$

CRBSI, Self-removal by accident

V. jug. dext. 102

CRBSI

AVF $\quad$ at the age of 5 years

Mean dwell time per catheter 212

Two girls, patients 2 and 3 tolerated the CVCs with only few complications and maximum dwell times of 698, and 595 days, respectively. Patients 1 and 4 suffered from repeated infections and dislocations, therefore, patient 4 received an AVF after eight months of LA. CRBSI: Catheter-Related Blood Stream Infection

In total, there were 12 (re)implantations of CVCs in four young children with hoFH treated with LA over three years. Patency of CVCs was most difficult to maintain within the first months. Six out of twelve 
CVCs were still in place after three months. Predominant reasons for early CVC loss were infection or accidental self-removal. Long-term patency rates were otherwise stable.

\section{Discussion}

This observational, retrospective study is the first evaluation of permanent CVCs as venous access for LA in young children with hoFH. Twelve CVC (re)implantations were necessary for four young children aged 3-7 years. As one boy suffered several CVC-related infections within the first year, the AVF was preferred. CVCs were used on three children until peripheral punctures were tolerated, at age 6 to 7 , and avoided AVF placement.

In this study, children aged 3 to 5 started LA with CVCs as venous access. In a publication from 2017, LA initiation depended on the patient's tolerance of peripheral venous puncture; therefore the procedure was never performed before the age of 6 years [28]. A systematic review by Luirink et al. recently highlighted the importance of starting LA as early as possible. Of the 123 analyzed patients with hoFH, $17 \%$ sustained a cardiovascular event before accessing LA treatment aged between 5 to 15 . The average age at the start of LA was 9.3 years, with only $12 \%$ of patients undergoing the procedure before age 5[14]. De facto, performing LA on young children is challenging because of small peripheral vessels raising technical problems related to venous puncture, low blood flow, and low blood volume $[14,29]$.

Anxiety and emotional distress can affect patients' compliance with LA treatment. After several peripheral punctures attempts to allow a 7-year-old girl (patient 3) more sporting activities, especially in the summer, such as swimming, her severe anxiety led to the decision to opt for a CVC. Nine months later, with intensive psychological support, and a better understanding of the illness, the child tolerated peripheral punctures and the CVC was electively removed. Psychological factors appear to be a concern even with the medical and nursing staff expertise, extensive psychological support, and local anesthesia.

In this small study cohort, infections and accidental self-removal were the most frequent complications. In summary, seven systemic infections ( 3 sepsis, 4 CRBSI) occurred in four children with hoFH. The cumulative CRBSI rate was 2.8 per 1,000 CVC days. Significantly more than previously published by Pinon et al., who reported a rate of 0.46 per $1,000 \mathrm{CVC}$ days in a pediatric cohort of oncological patients aged up to 20 (mean age 7.3 years) receiving long-term medication [24]. Former studies confirmed that young children are at increased risk of CVC infections $[22,25]$ and that long-term use of indwelling CVCs is a risk factor for CRBSI [30].

We observed frequent systemic and local infections in our cohort shortly after CVC implantation, thus the need for a central catheter replacement. Six out of twelve CVCs were still in situ after three months, resulting in a cumulative short-term duration rate of $50 \%$. Cesaro et al. reported that young age (under 5 years) is a predictor for premature CVC removal, particularly in the first two months after implantation [25]. 
Half of the CVCs were lost by accidental self-removal and needed catheter reimplantation. Estimates of dislocation rates in the literature are $31 \%$ of all complications associated with CVCs in children [24]. Young age is a known risk factor for dislocations $[22,24,31]$ which mainly occur early after implantation and may also be caused by incomplete cauterization of the cuff leading to poor adhesion [24].

Despite CVCs being the leading cause of thrombotic events in children, no catheter occlusion or thrombosis were observed in this study [32,33]. Most research on CVCs in pediatric cohorts assessed children with cancer; hence the prothrombotic state associated with hemato-oncological diseases could explain the higher rate of thrombosis in the literature [23-25]. However, apheresis influences coagulation and perhaps prevents thrombosis [36].

A severe long-term effect of repeated CVC insertions is vascular occlusion $[25,26]$. Regular doppler monitoring of supra-aortic blood flow revealed the absence of occlusions in the four children with hoFH.

Several studies proved that LA treatment promotes xanthoma regression, plaque stabilization, and even vascular regeneration $[2,14,34]$. We observed regression of xanthoma and no increase in supra-aortic IMT in our cohort.. Only one girl (patient 1) showed a slight IMT increase and developed a vascular plaque formation in the left internal carotid artery during the three years of LA, potentially resulting from frequent treatment interruptions due to the patients' non-compliance, catheter removal, or infections. Notably, after accidental CVC self-removal occurred in the second year of LA, only nine out of 21 LA sessions could be completed due to failure or intolerance to peripheral venous access.

While early-onset LA prevents premature cardiovascular events, obtaining venous access in young children may be problematic. There are three options for venous access, including peripheral venous punctures, forearm arteriovenous fistula (AVF), or permanent central venous catheters. Small-caliber veins, stress, and anxiety exclude peripheral venous punctures in younger children. The AVF procedure requires the vascular specialist to connect very small vessels and minimize the risk associated with the AV fistula, while the patient should be able to withstand the punctures during dialysis sessions[27, 35]. Moreover, AVFs in FH patients seem to be associated with a higher risk of vascular diseases [20, 37-39]. Central venous catheters avoid repeated treatments involving needles insertion and allow some movements during the LA procedure. On the other hand, the high risk of infections, dislocations, and other complications indicate a well-considered use, as described for hoFH children needing regular LA.

A limitation of this study may be the small sample size, although epidemiological data estimate the frequency of hoFH to be 1:160,000-1:300,000, categorizing the disease as rare [1]. Therefore, it is relevant and valuable to describe pros and cons of CVCs used in young hoFH children to enable LA early on, as the only effective therapy.

\section{Conclusions}

We can conclude that although central venous catheters are an option to start LA in young children with severe hoFH, this type of access needs careful management to avoid infections and dislocations and 
should only be used when urgency overrules contraindications. Moreover, it is essential to describe the pros and cons of CVCs in the youngest hoFH population to enable LA at an early stage as the only effective therapy. Delaying the start of LA exposes patients to premature cardiovascular events already in early childhood. Ultimately, AVFs can be an alternative to CVCs; however, the possibility to perform venipuncture earlier in life, subject to the patient's cooperation and vessel diameter, is preferable.

More data on venous access options for LA in early childhood is necessary to adequately assess the advantages and disadvantages of different approaches and guide clinical decision-making for the individual patient.

\section{Declarations}

\section{Ethics approval and consent to participate}

The study protocol was approved by the ethics committee of the Medical University of Vienna (No. 1638/2019) and conducted according to the Helsinki declaration guidelines. The need for written consent was waived because of the retrospective nature of the study and is thus deemed unnecessary according to national regulations.

\section{Consent for publication}

Not applicable.

\section{Availability of data and materials}

The datasets used and analyzed during the current study are available from the corresponding author on reasonable request.

\section{Competing interests}

The authors declare no competing interest.

\section{Funding}

No funding to disclose.

\section{Authors' contributions}

J.L. and S. G.P. planned the study concept, interpreted data, and drafted the manuscript. A.W. interpreted data and critically revised the manuscript. K.A. and M.B. reviewed and interpreted data on behalf of the apheresis unit. A. W.-E. reviewed and interpreted IMT measurements. J.L., N.-K. W., C. d. G. and R.G. acquired clinical data at the Outpatient Clinic for Pediatric Obesity and Dyslipidemia. All authors approved the final version of the manuscript.

\section{Acknowledgments}


We thank the staff at the outpatient clinic for lipid metabolism disorders at the Department of Pediatrics and Adolescent Medicine, Medical University Vienna, for their support. We acknowledge the support of the Scientific Publishing Service provided by P. Voitl and E. Tomasco, on behalf of the Austrian Pediatric Society.

\section{References}

1. Wiegman, A, Gidding, SS, Watts, GF, et al., Familial hypercholesterolaemia in children and adolescents: gaining decades of life by optimizing detection and treatment, European heart journal, 2015;36:2425-2437.

2. Cuchel, M, Bruckert, E, Ginsberg, HN, et al., Homozygous familial hypercholesterolaemia: new insights and guidance for clinicians to improve detection and clinical management. A position paper from the Consensus Panel on Familial Hypercholesterolaemia of the European Atherosclerosis Society, European heart journal, 2014;35:2146-2157.

3. Sijbrands, EJ, Nieman, K and Budoff, MJ, Cardiac computed tomography imaging in familial hypercholesterolaemia: implications for therapy and clinical trials, Current opinion in lipidology, 2015;26:586-592.

4. Stefanutti, C, Morozzi, C, Di Giacomo, S, et al., Management of homozygous familial hypercholesterolemia in real-world clinical practice: A report of 7 Italian patients treated in Rome with lomitapide and LA, Journal of clinical lipidology, 2016;10:782-789.

5. Martin, AC, Gidding, SS, Wiegman, A, et al., Knowns and unknowns in the care of pediatric familial hypercholesterolemia, Journal of lipid research, 2017;58:1765-1776.

6. Stefanutti, C and Thompson, GR, LA in the management of familial hypercholesterolaemia: historical perspective and recent advances, Current atherosclerosis reports, 2015;17:465.

7. Dumic, M, Uroic, AS, Francetic, I, et al., [Three-year-old boy-a homozygote for familiar hypercholesterolemia], Lijecnicki vjesnik, 2007;129:130-133.

8. Gautschi, M, Pavlovic, M and Nuoffer, JM, Fatal myocardial infarction at 4.5 years in a case of homozygous familial hypercholesterolaemia, JIMD reports, 2012;2:45-50.

9. Widhalm, K, Binder, CB, Kreissl, A, et al., Sudden death in a 4-year-old boy: a near-complete occlusion of the coronary artery caused by an aggressive low-density lipoprotein receptor mutation (W556R) in homozygous familial hypercholesterolemia, The Journal of pediatrics, 2011;158:167.

10. Macchiaiolo, M, Gagliardi, MG, Toscano, A, et al., Homozygous familial hypercholesterolaemia, Lancet, 2012;379:1330.

11. France, M, Rees, A, Datta, D, et al., HEART UK statement on the management of homozygous familial hypercholesterolaemia in the United Kingdom, Atherosclerosis, 2016;255:128-139.

12. Kroon, AA, van't Hof, MA, Demacker, PN, et al., The rebound of lipoproteins after LDL-apheresis. Kinetics and estimation of mean lipoprotein levels, Atherosclerosis, 2000;152:519-526. 
13. Stefanutti, C and Julius, U, LA: state of the art and novelties, Atherosclerosis. Supplements, 2013;14:19-27.

14. Luirink, IK, Determeijer, J, Hutten, BA, et al., Efficacy and safety of LA in children with homozygous familial hypercholesterolemia: A systematic review, Journal of clinical lipidology, 2019;13:31-39.

15. Moriarty, PM, LDL-apheresis therapy, Current treatment options in cardiovascular medicine, 2006;8:282-288.

16. Thompson, GR, LDL apheresis, Atherosclerosis, 2003;167:1-13.

17. Wooster, M, Wilson, $R$, Shames, $M$, et al., Arteriovenous access does not perform as well for plasmapheresis, The journal of vascular access, 2017;18:144-147.

18. Ipe, TS and Marques, MB, Vascular access for therapeutic plasma exchange, Transfusion, 2018;58 Suppl 1:580-589.

19. Larson, J, Adrift in the world of therapeutic apheresis: One center's experience in using arterio-venous fistulas during apheresis procedures, Transfusion and apheresis science: official journal of the World Apheresis Association : official journal of the European Society for Haemapheresis, 2016;55:264265.

20. Doherty, DJ, Pottle, A, Malietzis, G, et al., Vascular access in LA: a retrospective analysis from the UK's largest LA centre, The journal of vascular access, 2018;19:52-57.

21. Lee, M, Barr, J, Kribs, S, et al., Strategies to reduce line infections in a small child with homozygous familial hypercholesterolaemia who cannot yet receive LDL apheresis, BMJ case reports, 2017;2017.

22. de Jonge, RC, Polderman, $\mathrm{KH}$ and Gemke, RJ, Central venous catheter use in the pediatric patient: mechanical and infectious complications, Pediatric critical care medicine: a journal of the Society of Critical Care Medicine and the World Federation of Pediatric Intensive and Critical Care Societies, 2005;6:329-339.

23. Fratino, G, Molinari, AC, Parodi, $S$, et al., Central venous catheter-related complications in children with oncological/hematological diseases: an observational study of 418 devices, Annals of oncology: official journal of the European Society for Medical Oncology, 2005;16:648-654.

24. Pinon, M, Bezzio, S, Tovo, PA, et al., A prospective 7-year survey on central venous catheter-related complications at a single pediatric hospital, European journal of pediatrics, 2009;168:1505-1512.

25. Cesaro, S, Corro, R, Pelosin, A, et al., A prospective survey on incidence and outcome of Broviac/Hickman catheter-related complications in pediatric patients affected by hematological and oncological diseases, Annals of hematology, 2004;83:183-188.

26. Merouani, A, Lallier, M, Paquet, J, et al., Vascular access for chronic hemodialysis in children: arteriovenous fistula or central venous catheter?, Pediatric nephrology (Berlin, Germany), 2014;29:2395-2401.

27. Baracco, R, Mattoo, T, Jain, A, et al., Reducing central venous catheters in chronic hemodialysis-a commitment to arteriovenous fistula creation in children, Pediatric nephrology (Berlin, Germany), 2014;29:2013-2020. 
28. Widhalm, K, Benke, IM, Fritz, M, et al., Homozygous familial hypercholesterolemia: Summarized case reports, Atherosclerosis, 2017;257:86-89.

29. Stefanutti, C, Notarbartolo, A, Colloridi, V, et al., LDL apheresis in a homozygous familial hypercholesterolemic child aged 4.5, Artif Organs, 1997;21:1126-1127.

30. Rosado, V, Romanelli, RM and Camargos, PA, Risk factors and preventive measures for catheterrelated bloodstream infections, J Pediatr (Rio J), 2011;87:469-477.

31. Polderman, KH and Girbes, AJ, Central venous catheter use. Part 1: mechanical complications, Intensive Care Med, 2002;28:1-17.

32. Journeycake, JM and Buchanan, GR, Thrombotic complications of central venous catheters in children, Curr Opin Hematol, 2003;10:369-374.

33. Neunert, CE, Miller, KL, Journeycake, JM, et al., Implantable central venous access device procedures in haemophilia patients without an inhibitor: systematic review of the literature and institutional experience, Haemophilia, 2008;14:260-270.

34. Schuff-Werner, P, Fenger, S and Kohlschein, P, Role of I in changing times, Clin Res Cardiol Suppl, 2012;7:7-14.

35. Bourquelot, P, [Vascular access for haemodialysis], Nephrol Ther, 2009;5:239-248.

36. Knisel W, Di Nicuolo A, Pfohl M, et al. Different effects of two methods of low-density lipoprotein apheresis on the coagulation and fibrinolytic systems. J Intern Med, 1993;234(5):479-87.

37. De Marchi, S, Falleti, E, Giacomello, R, et al., Risk factors for vascular disease and arteriovenous fistula dysfunction in hemodialysis patients, J Am Soc Nephrol, 1996;7:1169-1177.

38. Lappegård, KT and Hovland, A, Side effects in LDL apheresis: types, frequency and clinical relevance, Clinical Lipidology, 2011;6:717-722.

39. Beigel, R and Beigel, Y, Homozygous familial hypercholesterolemia: long term clinical course and plasma exchange therapy for two individual patients and review of the literature, J Clin Apher, 2009;24:219-224. 